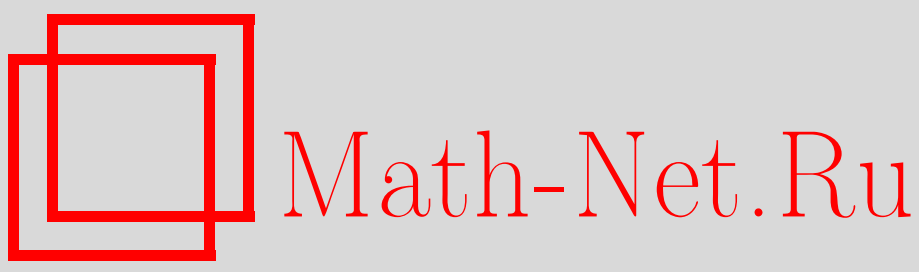

П. В. Семенов, Теоремы о неподвижной точке при контролируемом отказе от выпуклости значений многозначного отображения, Матем. сб., 1998, том 189, номер 3, 141-160

DOI: https://doi.org/10.4213/sm314

Использование Общероссийского математического портала Math-Net.Ru подразумевает, что вы прочитали и согласны с пользовательским соглашением

http: //www.mathnet.ru/rus/agreement

Параметры загрузки:

IP : 54.198 .64 .247

26 апреля 2023 г., 13:21:24 
УДК 513.83

\author{
П.В. Семенов
}

\title{
Теоремы о неподвижной точке при контролируемом отказе от выпуклости значений многозначного отображения
}

\begin{abstract}
В настоящей работе исследуется вопрос о том, в какой степени можно отказаться от выпуклости значений многозначных отображений в классических теоремах Какутани, Боннебласта-Карлина, Гликсберга о неподвижных точках. Для ответа на этот вопрос с каждым замкнутым подмножеством $P$ банахова пространства ассоциируется некоторая числовая функция $\alpha_{P}:(0, \infty) \rightarrow[0, \infty)$, называемая функцией невыпуклости множества $P$. Чем ближе функция невыпуклости $\alpha_{P}$ к нулю, тем "вьпуклее" становится множество $P$. Равенство $\alpha_{P} \equiv 0$ эквивалентно выпуклости $P$. В работе доказаны селекционные, аппроксимационные теоремы и теоремы о неподвижных точках для многозначных отображений $F$ конечномерных и бесконечномерных паракомпактов с заменой условия $\alpha_{F(x)} \equiv 0$ на условия типа " $\alpha_{F(x)}$ меньше единицы". Сравниваются различные формализации последнего условия и доказана топологическая устойчивость такого типа ограничений.
\end{abstract}

Библиография: 25 названий.

\section{Введение}

Классическая теорема Брауэра о неподвижной точке непрерывного отображения двумерного диска в себя была обобщена на случай отображений с бесконечномерной замкнутой (компактной) выпуклой областью определения Шаудером [1] (соответственно Тихоновым). Для выпуклозначных отображений конечномерного пространства существование неподвижных точек доказал Какутани [2], а в случае бесконечномерных областей определения - Боннебласт и Карлин (многозначная теорема Шаудера) [3] и Гликсберг (многозначная теорема Тихонова или же бесконечномерная теорема Какутани) [4]. Подробные сведения об использовании топологических методов в теории неподвижных точек многозначных отображений можно найти в статье [5]; см. также обзор [6].

В настоящей работе исследуется вопрос о том, в какой степени можно отказаться от выпуклости значений многозначных отображений в указанных теоремах о неподвижных точках. Для ответа на этот вопрос требуется рассмотреть некий объект, “измеряющий” уклонение от вьпуклости замкнутых подмножеств нормированных пространств. В качестве таких объектов мы рассматриваем здесь числовые функции $\alpha:(0, \infty) \rightarrow[0, \infty)$.

Работа вьполнена при поддержке Российского фонда фундаменталњных исследований (грант № 96-01-01166а). 
Для замкнутого непустого подмножества $P \subset E$ нормированного пространства $(E,\|\cdot\|)$ и для открытого шара $D \subset E$ число

$$
\Delta(D, P)=\sup \{\operatorname{dist}(x, P) \mid x \in \operatorname{conv}(D \cap P)\}
$$

естественно измеряет "абсолютную" невыпуклость множества $P$ внутри шара $D$, а отношение $\Delta(D, P) / r$ есть "относительная" невыпуклость $P$ внутри $D$. Здесь, как обычно, $\operatorname{conv}(D \cap P)$ - выпуклая оболочка пересечения $D \cap E$ и $\operatorname{dist}(x, P)=$ $\inf \{\|x-p\| \mid p \in P\}$.

ОПРЕДЕЛЕНИЕ 1. Функиией невыпуклости $\alpha_{P}(\cdot)$ замкнутого непустого подмножества $P$ нормированного пространства $E$ называется функция, которая каждому числу $r>0$ ставит в соответствие число

$$
\alpha_{P}(r)=\sup \{\Delta(D, P) / r \mid D-\text { открытый шар радиуса } r\} .
$$

Чем ближе функция невыпуклости $\alpha_{P}$ к нулю, тем "вьпуклее" становится множество $P$. Равенство $\alpha_{P} \equiv 0$ эквивалентно выпуклости $P$. Ясно, что значения функции невыпуклости всегда лежат в отрезке $[0,2]$, а если норма в пространстве $E$ задана скалярным произведением, то в отрезке $[0,1]$. Можно показать, что последнее свойство характеризует (в размерности большей двух) пространства со скалярным произведением.

Контролируемый отказ от вьпуклости в настоящей работе состоит в том, что мы рассматриваем множества, функции невыпуклости которых, грубо говоря, меньше единицы. Для формального определения последнего понятия ассоциируем с каждой функцией $\alpha:(0, \infty) \rightarrow[0, \infty)$ некоторую функциональную последовательность $q^{n}=q_{\alpha}^{n}:(0, \infty) \rightarrow[0, \infty), n \in \mathbb{N}$, по правилу:

$$
q^{0}(t)=t, q^{1}(t)=\alpha(t) t, \ldots, q^{n+1}(t)=\alpha\left(q^{n}(t)\right) q^{n}(t), \ldots
$$

Другими словами, $\left\{q_{\alpha}^{n}\right\}$ есть геометрическая прогрессия со знаменателем $\alpha$. Если при этом $\alpha\left(q^{n}(t)\right)=0$ для некоторого $n$, то по определению положим $q^{n+2}(t)=$ $q^{n+3}(t)=\cdots=0$.

ОПРЕДЕЛЕНИЕ 2. Будем говорить, что функция $\alpha:(0, \infty) \rightarrow[0, \infty) G$-суммируем $a$, если функциональный ряд $\sum_{n=0}^{\infty} q_{\alpha}^{n}(\cdot)$ поточечно сходится на $(0, \infty)$; сумму этого ряда обозначим $S_{\alpha}(t)$.

Обозначим через $\alpha^{+}$функцию $\alpha^{+}(s)=\varlimsup_{t \rightarrow s+0} \alpha(t), s>0$. Естественно также обозначить через $\alpha^{+0}$ функцию $\alpha^{+0}(s)=\varlimsup_{i \rightarrow s+0} \alpha(t), s \geqslant 0$.

ПРЕДЛОЖЕНИЕ 3. Рассмотрим следующие свойства числовой функиии $\alpha:(0, \infty) \rightarrow[0,1):$

(1) функция $\alpha^{+0}$ меньше единицы;

(2) функция $\alpha$ G-суммируема;

(3) функиия а имеет G-суммируемую строгую мажсоранту;

(4) функиия $\alpha^{+}$имеет G-суммируемую строгую мажоранту. 
Тогда справедливы импликации (1) $\Rightarrow(2),(1) \Rightarrow(3),(1) \Rightarrow(4)$, неизвестна справедливость импликации $(4) \Rightarrow(3)$, а остальные импликации ложны.

Типичный пример вьполнимости всех свойств (1)-(4) из предложения 3 - монотонно возрастающие функции $\alpha:(0, \infty) \rightarrow[0,1)$ или же функции, мажорируемые таковыми. В случае (3) будем говорить, что функция $\alpha$ заметно меншше единицы, и использовать обозначение $\alpha<1-0$. В случае (4) будем говорить, что функция $\alpha$ меньше единицы справа.

Следующая теорема является основной в данной работе и является невьпуклозначной версией теоремы Гликсберга.

ТЕОРема 4. Пусть компактное подмножество К банахова пространства $(B,\|\cdot\|)$ является абсолютныц окрестностным ретрактом и обладает свойством неподвижной точки для однозначньх отображений в себя. Пусть $F: K \rightarrow K$ - полунепрерывное сверху компактнозначное отображение, и пусть для каждого $x \in K$ функиия невыпуклости множества $F(x)$ заметно меньше единицы. Тогда $F$ имеет неподвижную точку, т.е. имеется точка $x_{0} \in K$ такая, что $x_{0} \in F\left(x_{0}\right)$.

Невыпуклозначная версия теоремы Боннебласта-Карлина состоит в том, что полунепрерывное сверху отображение $F: A \rightarrow A$ замкнутого подмножества $A$ банахова пространства $B$ имеет неподвижную точку, как только к замыканию $K=\operatorname{clos}\{F(A)\}$ множества $F(A)=\bigcup\{F(a) \mid a \in A\}$ применима теорема 4.

Реально теорема 4 применима к любому абсолютному ретракту $K$. В частности, она применима к любому компакту $K$ с $\alpha_{K}<1-0$, как показывает следующая теорема 5 , в которой из оценки $\alpha_{P}<1-0$ выводится топологическая тривиальность множества $P$ и его аппроксимативная топологическая тривиальность.

Теорема 5. Если функция невыпуклости замкнутого подмножества $P$ банахова пространства $B$ заметно меньше единицы, то:

1) $P$ есть $U V R$-подмножество $B$, т.е. для любого $\varepsilon>0$ найдется $\delta>0$ такое, что существует ретракция $R_{\delta}: D_{\delta}(P) \rightarrow P \quad \delta$-окрестности множества $P$ на само множество $P$, которая внутри $\varepsilon$-окрестности $D_{\varepsilon}(P)$ гомотопна постоянному отображению $D_{\delta}(P)$ в себя.

2) Р есть абсолютный экстензор для паракомпактных пространств.

Теорема 4 является следствием теоремы 5 в силу аппроксимационной теоремы [7], [8], в которой наличие однозначных (графических) аппроксимаций многозначного отображения выводится из $U V$-свойств значений этого отображения. Теорема 5 , в свою очередь, есть следствие следуюшей селекционной теоремы 6.

Теорема 6. Пусть $\Phi: X \rightarrow B$ - полунепрерьвное снизу отображсение паракомпакта $X$ в банахово пространство $B$, и пусть существует заметно меньшая единицы мажсоранта семейства функиий невыпуклости всех значений $\Phi(x), x \in X$. Тогда существует непрерьвная однозначная селекиия отображения $\Phi$.

Для формулировки конечномерной версии теоремы 4 о неподвижной точке определим множество $\operatorname{conv}_{n+1}(A)$ как объединение всех выпуклых оболочек не более чем $(n+1)$-элементных подмножеств множества $A$. 
ОПРЕДЕЛЕНИЕ 7. Функиией невыпуклости $\alpha_{P}^{n}$ (в размерности $n$ ) замкнутого непустого подмножества $P$ нормированного пространства $E$ назьвается функция, которая каждому числу $r>0$ ставит в соответствие число

$$
\alpha_{P}^{n}(r)=\sup \left\{\Delta_{n+1}(D, P) / r \mid D-\text { открытый шар радиуса } r\right\}
$$

где

$$
\Delta_{n+1}(D, P)=\sup \left\{\operatorname{dist}(x, P) \mid x \in \operatorname{conv}_{n+1}(D \cap P)\right\} .
$$

Всюду ниже под размерностью dim нормального топологического пространства понимается его лебегова размерность.

ТеОрема 8. Пусть $\Phi: X \rightarrow B$ - полунепрерывное снизу отображсение n-мерного (в смысле $\operatorname{dim}$ ) паракомпакта $X$ в банахово пространство $B, u$ пусть существует меньшая единицы справа мажсоранта семейства функций невъпуклости (в размерности $n$ ) всех значений $\Phi(x), x \in X$. Тогда отображсение $\Phi$ имеет непрерывную однозначную селекиию.

Теорема 8 есть явный аналог теоремы 6 , но доказательство теоремы 8 принципиально отличается от доказательства теоремы 6 . Кратко говоря, объяснение этого несоответствия заключается в том, что переход от множества $A$ к множеству $\operatorname{conv} A$ дает выпуклое множество, т.е. "максимально" простое с топологической точки зрения множество. В то же время, переход от множества $A$ к множеству $\operatorname{conv}_{n+1} A$ улучшает глобальные топологические свойства, но может и ухудшать локальные топологические свойства. Например, множество $A$ может быть локально связным, а множество $\operatorname{conv}_{2} A$ не быть таковым. Видимо, по этой причине в теореме 8 приходится использовать наличие $G$-суммируемой мажоранты у функции $\alpha^{+}$, а не у функции $\alpha$, как в теореме 6 . Следствием селекционной теоремы 8 является следующий конечномерный аналог теоремы 4 о неподвижной точке.

ТЕОРема 9. Пусть компактное подмнохсество $K$ банахова пространства $(B,\|\cdot\|)$ является абсолютным ретрактом $u \operatorname{dim} K \leqslant n$. Пусть $F: K \rightarrow K-$ полунепрерывное сверху компактнозначное отображение, и пусть для каждого $x \in K$ функиия невыпуклости (в размерности $n$ ) множсества $F(x)$ меньше единищы справа. Тогда $F$ имеет неподвижсную точку.

Определение функции невьпуклости несколько напоминает определение модуля равномерной вьпуклости сферы банахова пространства: мы двигаем по множеству вершины симплекса фиксированного чебышевского радиуса и смотрим насколько точки симплекса удалены при этом от самого множества. Более явньм образом, понятие функции невыпуклости обобщает понятие $\alpha$-паравыпуклых множеств, введенное Майклом в [9]. Так называются множества, функция невыпуклости которых мажорируется на всем луче $(0, \infty)$ константой $0 \leqslant \alpha<1$.

Явное вычисление или же нахождение хорошей верхней оценки функции невыпуклости конкретного множества - вещь достаточно трудоемкая. Положительные результаты имеются для поперечных возмущений выпуклых множеств, т.е. для графиков непрерывных функций с выпуклыми областями определения [10]-[12] и для продольных возмушений выпуклых множеств, т.е. для букетов выпуклых множеств [13]. Один из центральных результатов настоящей работы состоит в некоторой устойчивости семейства множеств, у которых функции невыпуклости заметно меньше единицы. 
ТЕОРЕМа 10. Пусть данъ равномерно выпуклое банахово пространство $(B,\|\cdot\|)$, функиия $\alpha:(0, \infty) \rightarrow[0,1)$ заметно меньиая единиць и число $\varepsilon>0$. Тогда существует функиия $\beta:(0, \infty) \rightarrow[0,1)$ заметно меньиая единиць такая, что для любого множества $P$ с $\alpha_{P} \leqslant \alpha$ функиия невыпуклости замкнутой є-окрестности $\bar{D}_{\varepsilon}(P)$ множсества $P$ не превосходит функиии $\beta$.

Кратко говоря, $\varepsilon$-окрестности хороших множеств тоже не плохие. Следующий пример показывает, что невыпуклость сколь угодно малых $\varepsilon$-окрестностей множества, вообще говоря, может существенно отличаться от невыпуклости самого множества, т.е. в теореме 10 при фиксированном множестве $P$ и при $\varepsilon \rightarrow 0$ функцию $\beta$ нельзя считать сходящейся к функции $\alpha_{P}$.

ПРЕДЛОЖЕНИЕ 11. Для любого числа $0 \leqslant q<1$ найдется число $p \in(q, 1)$ и найдется q-паравыпуклый компакт $K \subset \mathbb{R}^{2}$, который имеет сколь угодно малье не р-паравыпуклье замкнутые окрестности.

Следствием теоремы 10 является следующая общая аппроксимационная теорема 12. Напомним, что (әрафической) $\varepsilon$-аппроксимацией многозначного отображения $H: X \rightarrow Y$ метрических пространств $(X, \rho),(Y, d)$ называется однозначное непрерывное отображение $h_{\varepsilon}: X \rightarrow Y$ такое, что его график $\Gamma\left(h_{\varepsilon}\right)$ лежит в $\varepsilon$-окрестности графика $\Gamma(H)$ относительно тах-метрики в декартовом произведении $X \times Y$.

Tеорема 12. Пусть $(X, d)$ - метрическое пространство, $\operatorname{dim} X \leqslant n$, пусть $F: X \rightarrow B$ - полунепрерывное сверху компактнозначное отобрахсение в равномерно выпуклое банахово пространство $(B,\|\cdot\|)$, и пусть для кажсдого $x \in X$ функция невыпуклости (в размерности $n$ ) мнохсества $F(x)$ заметно меньше единицы. Тогда F имеет непрерывные однозначные $\varepsilon$-аппроксимации для любого $\varepsilon>0$.

Теоремы 4, 6, 8, 12 настоящей работы являются метрическими невыпуклозначными аналогами классических теорем для выпуклозначных отображений. В случае полиэдральной области определения $X$ гомологический и соответственно гомотопический подходы к доказательству соответствующих теорем о невыпуклозначных отображениях развиты в работах [14], [15].

Ниже следующий текст организован так. В $\S 1$ собраны технические утверждения о соотношениях между различными уточнениями понятия “функция меншше единицы", т.е. доказано предложение 3 . В $\S 2$ доказаны бесконечномерные теоремы 4, 5, 6. В следуюшем $\S 3$ доказаны их конечномерные аналоги: теоремы 8 и 9. Наконец, в заключительном $\S 4$ доказаны теорема 10 и теорема 12 , являющаяся конечномерным невыпуклозначным аналогом известной аппроксимационной теоремы Челлины [16]. Стандартные сведения о многозначных отображениях и их селекциях см. в [17], [18], [5], [6].

\section{§1. Функции, мажорируемые $G$-суммируемыми}

Отметим, что условие $\overline{\lim }_{t \rightarrow s+0} \alpha(t)<1, s>0$, естественно возникает в теории неподвижных точек многозначных обобщенных сжатий. Более точно, с 1983 года открыта проблема [19] о достаточности этого условия для наличия неподвижных точек у $\alpha$-сжатий полных метрических пространств $M$, т.е. отображений 
$C: M \rightarrow M$ таких, что

$$
H_{\rho}(C(x), C(y)) \leqslant \alpha(\rho(x, y)) \cdot \rho(x, y), \quad x, y \in M,
$$

где $H_{\rho}$ - хаусдорфова метрика между подмножествами пространства $M$, ассоциированная с данной метрикой $\rho$ самого пространства $M$. В работе [20] показано, что добавление к этому условию случая $s=0$, т.е. в точности условие (1) предложения 3 , дает положительное решение данной проблемы.

В то же время условие $G$-суммируемости и, тем более, условие $\alpha<1-0$, существенно слабее условия $\alpha^{+0}<1$. В свою очередь, условие $G$-суммируемости не наследственно, т.е. сушествуют не $G$-суммируемые функции, которые мажорируются $G$-суммируемыми. Кроме того, $G$-суммируемьми могут быть и функции невьпуклости несвязных множеств. Именно по этой причине приходится в теоремах $4,5,6$ ослаблять $G$-суммируемость функций невьпуклости $\alpha_{F(x)}$ до условия, что $\alpha_{F(x)}$ заметно меньше единицы. Последнее условие очевидно уже наследственно: если $\beta<1-0$ и $\alpha \leqslant \beta$, то и $\alpha<1-0$.

ДоКАЗАТЕЛЬСТВо ПРЕДЛОЖЕНИЯ 3. Докажем импликации $(1) \Rightarrow(2)$ и (1) $\Rightarrow(3)$.

Если $\varlimsup_{t \rightarrow s+0} \alpha(t)$ менњше единицы при всех $s \geqslant 0$, то же самое верно и для функции $\beta=(1+\alpha) / 2, \beta>\alpha$. Докажем, что функция $\beta$ является $G$-суммируемой. $\Phi$ иксируем $t>0$ и рассмотрим числовую последовательность $\left\{q_{\beta}^{n}(t)\right\}=\left\{b_{n}\right\}$ :

$$
q^{0}(t)=t, q^{1}(t)=\beta(t) t, \ldots, q^{n+1}(t)=\beta\left(q^{n}(t)\right) q^{n}(t), \ldots
$$

Ясно, что последовательность $\left\{b_{n}\right\}$ строго убывает к некоторому числу $s \geqslant 0$. Из равенства $b_{n+1}=\beta\left(b_{n}\right) b_{n}$ следует, что $s=0$. Так как правый верхний предел функции $\beta$ в $s=0$ строго меньше единицы, то для некоторой константы $k<1$ при всех достаточно больших $n$ верно неравенство $\beta\left(b_{n}\right) \leqslant k$. Значит, начиная c некоторого номера числовой ряд $\sum b_{n}$ мажорируется суммой геометрической прогрессии $\sum k^{n}$.

$(1) \Rightarrow(4)$. Если функция $\gamma$ есть максимум функций $\alpha$ и $\alpha^{+}$, то $\gamma^{+0}<1$ и поэтому $\beta^{+0}<1$, где $\beta=(1+\gamma) / 2$ есть строгая мажоранта функции $\alpha^{+}$. По предыдущему доказательству функция $\beta G$-суммируема.

Контрпример к импликациям $(2) \Rightarrow(1)$ и $(4) \Rightarrow(1)$. Пусть $\alpha(\cdot)$ равна нулю вне точек $1 / n^{2}$ и $\alpha\left(1 / n^{2}\right)=n(n+1)$. Тогда для всех $t>0$ значения функции $q_{\alpha}^{1}(t)=$ $\alpha(t) t$ лежат вне последовательности $\left\{1 / n^{2}\right\}$ и поэтому числовой ряд $\sum q_{\alpha}^{n}(t)$ состоит из не более, чем двух слагаемых. Значит, функция $\alpha G$-суммируема, а е верхний предел в нуле равен единице. Кроме того, функция $\alpha^{+}$тождественно равна нулю и поэтому имеет $G$-суммируемую мажоранту.

Контрпример к импликации $(2) \Rightarrow(3)$ (значит, и к $(2) \Rightarrow(1))$. Пусть на $\left(0, a_{1}\right]$ функция $\alpha$ равна константе $k<1$, а на полуинтервалах $\left(a_{n}, a_{n+1}\right]$ для функции $\alpha$ верно неравенство $\alpha(t) \leqslant a_{n} / t$, где $0<a_{1}<a_{2}<a_{3}<\ldots$ - некоторая фиксированная последовательность. Тогда для $t>0$ при некотором $m$ верно включение $t \in\left(a_{m}, a_{m+1}\right]$ и, значит, значения функции $q_{\alpha}^{1}(t)=\alpha(t) t$ лежат на $\left(a_{k}, a_{k+1}\right]$ для некоторого $k<m$. Следовательно, значения функции $q_{\alpha}^{2}(t)=\alpha\left(q_{\alpha}^{1}(t)\right) \cdot q_{\alpha}^{1}(t)$ лежат на $\left(a_{p}, a_{p+1}\right]$ для некоторого $p<k<m$ и т.д. Поэтому числовой ряд $\sum q_{\alpha}^{n}(t)$, начиная с $n=m$, мажорируется рядом $\sum k^{n}$. Значит, функция $\alpha G$-суммируема, 
а в точках $s=a_{n}$ верхние пределы у $\alpha$ справа могут равняться 1 . Этот пример весьма типичен в том смысле, что для любого конечного подмножества $P$ банахова пространства $(B,\|\cdot\|)$ функция невыпуклости $\alpha_{P}$ имеет в точности описанный вид для $k=0$ и для некоторой последовательности $0<a_{1}<a_{2}<a_{3}<\ldots$ и при этом на полуинтервалах $\left(a_{n}, a_{n+1}\right]$ функция $\alpha_{P}$ равна $a_{n} / t$. Если бы у $G$-суммируемой функции $\alpha_{P}$ сушествовала бы $G$-суммируемая мажоранта, то по теореме 5 множество $P$ было бы стягиваемо, а это не так.

Контрпример к импликациям $(2) \Rightarrow(4)$ и $(3) \Rightarrow(4)$. Пусть, как и выше, на $\left(0, a_{1}\right]$ функция $\alpha$ равна константе $k<1$, а на полуинтервалах $\left(a_{n}, a_{n+1}\right]$ для функции $\alpha$ верно неравенство $\alpha(t) \leqslant a_{n} / t$, где $0<a_{1}<a_{2}<\ldots$ - некоторая фиксированная последовательность. Тогда $\alpha$ строго мажорируется $G$-суммируемой функцией невьпуклости подходящего конечного множества, а функция $\alpha^{+}$не имеет такой мажоранты просто потому, что в некоторых точках она равна единице.

Контрпример к импликации (3) $\Rightarrow(2)$ (значит, и к (3) $\Rightarrow(1)$ ). Фиксируем монотонно убывающую к единице последовательность $a_{n}$ с $a_{1}=2$ и определим функцию $\alpha:(0, \infty) \rightarrow[0,1)($ функцию $\beta:(0, \infty) \rightarrow[0,1))$ равной $2 / t($ равной $3 / t)$ при $t \geqslant 4$, равной $1 / 5$ (равной $1 / 4)$ при $t<4$ и $t \notin\left\{a_{n}\right\}$ и равной $a_{n+1} / a_{n}$ (равной $\left.\left(1+a_{n+1} / a_{n}\right) / 2\right)$ при $t=a_{n}$. Ясно, что $\beta>\alpha$. Докажем, что функция $\alpha$ не $G$-суммируема. При $t \geqslant 4$ имеем $q_{\alpha}^{1}(t)=t \cdot \alpha(t)=2=a_{1}, q_{\alpha}^{2}(t)=\alpha\left(q_{\alpha}^{1}(t)\right) \cdot q_{\alpha}^{1}(t)=$ $a_{1} \cdot \alpha\left(a_{1}\right)=a_{2}, \ldots, q_{\alpha}^{n}(t)=a_{n}>1, \ldots$. Поэтому $S_{\alpha}(t)=\infty$. Докажем, что функция $\beta$ G-суммируема. При $t \geqslant 4$ имеем $q_{\beta}^{1}(t)=t \cdot \beta(t)=3$ и $q_{\beta}^{2}(t)=$ $\beta\left(q_{\beta}^{1}(t)\right) \cdot q_{\beta}^{1}(t)=3 \cdot \beta(3)=3 / 4<1$. Значит, $q_{\beta}^{n+1}(t)=q_{\beta}^{n}(t) / 4$ при $n \geqslant 2$ и поэтому $S_{\beta}(t)<\infty$. Если $t<4$ и $t \notin\left\{a_{n}\right\}$, то $q_{\beta}^{1}(t)=t \cdot \beta(t)=t / 4<1$ и, значит, как и выше, $q_{\beta}^{n+1}(t)=q_{\beta}^{n}(t) / 4$ при $n \geqslant 2$. Если же $t=a_{n}$, то $a_{n+1} / a_{n}<\beta(t)<1$ и поэтому $a_{n+1}<q_{\beta}^{1}(t)<a_{n}$, т.е. $q_{\beta}^{1}(t) \notin\left\{a_{n}\right\}$. Значит, $S_{\beta}\left(q_{\beta}^{1}(t)\right)<\infty$ и $S_{\beta}(t)=t+S_{\beta}\left(q_{\beta}^{1}(t)\right)<\infty$, т.е. функция $\beta$ есть $G$-суммируемая строгая мажоранта не $G$-суммируемой функции $\alpha$.

Контрпример к импликации $(4) \Rightarrow(2)$. Фиксируем монотонно убывающую к нулю последовательность $a_{n}$, и пусть $\alpha(\cdot)$ равна нулю вне точек $a_{n}$, а $\alpha\left(a_{n}\right)=$ $a_{n+1} / a_{n}$. Тогда функция $\alpha^{+}$тождественно равна нулю и поэтому имеет $G$-суммируемую мажоранту. В то же время $q_{\alpha}^{n}\left(a_{1}\right)=a_{n}$ и поэтому $S_{\alpha}\left(a_{1}\right)=\sum a_{n}$. Остается взять $a_{n}$ так, чтобы последний ряд расходился. Предложение 3 доказано.

Прокомментируем сложность опровержения импликации $(4) \Rightarrow(3)$. Если $\alpha$ мажорирует функцию невыпуклости некоторого множества $P$, то из условия (4), по теореме 8 , следует, что $P$ есть абсолютный экстензор для всех конечномерных паракомпактов. А невыполнения условия (3) можно было бы по теореме 6 добиться, если бы $P$ не было абсолютным экстензором для всех паракомпактов. Примеры таких множеств существуют [21], но явная оценка функций невыпуклости конкретно для них затруднительна.

\section{§2. Контролируемый отказ от выпуклости. Бесконечномерный случай}

ДОКАЗАТЕЛЬСТВО ТЕОРЕМЫ 6. СЛучай 1. Начнем со случая, когда в условиях этой теоремы для некоторой константы $r>0$ найдется непрерьвная однозначная 
$r$-селекция $g: X \rightarrow B$ отображения $\Phi: X \rightarrow B$, т.е.

$$
\operatorname{dist}(g(x), \Phi(x))<r, \quad x \in X .
$$

Мы хотим, стартуя с этой $r$-селекции, найти некоторую непрерывную однозначную селекцию $\varphi: X \rightarrow B$ отображения $\Phi: X \rightarrow B, \varphi(x) \in \Phi(x)$ при всех $x \in X$, и оценить насколько при таком улучшении мы удалимся от первоначального положения. Итак, положим $\varphi_{0}=g$ и

$$
\Phi_{1}(x)=\overline{\operatorname{conv}}\left\{D\left(\varphi_{0}(x), r\right) \cap \Phi(x)\right\}, \quad x \in X .
$$

Отображение $\Phi_{1}: X \rightarrow B$ имеет непустые замкнутые вьпуклые значения и полунепрерывно снизу в силу полунепрерывности снизу отображения $\Phi$ и непрерывности однозначного отображения $\varphi$. По классической теореме Майкла [18] отображение $\Phi_{1}$ имеет непрерывную однозначную селекцию, скажем $\varphi_{1}$. Тогда при всех $x \in X$ верны неравенства $\left\|\varphi_{1}(x)-\varphi_{0}(x)\right\| \leqslant r=q_{\alpha}^{0}(r)$ и в силу определения 1 функции невьпуклости множества $\Phi(x)$

$$
\operatorname{dist}\left(\varphi_{1}(x), \Phi(x)\right) \leqslant \alpha_{\Phi(x)}(r) \cdot r<\alpha(r) \cdot r=r_{1}=q_{\alpha}^{1}(r)
$$

В последнем неравенстве функция $\alpha$ есть $G$-суммируемая строгая мажоранта функций невыпуклости множеств $\Phi(x), x \in X$. Если непрерывные однозначные отображения $\varphi_{0}, \varphi_{1}, \ldots, \varphi_{n}$ из $X$ в $B$ уже построены так, что при всех $x \in X$ верны неравенства

$$
\begin{aligned}
\left\|\varphi_{n}(x)-\varphi_{n-1}(x)\right\| & \leqslant r_{n-1}=q_{\alpha}^{n-1}(r), \\
\operatorname{dist}\left(\varphi_{n}(x), \Phi(x)\right) & <r_{n}=q_{\alpha}^{n}(r),
\end{aligned}
$$

то положим

$$
\Phi_{n+1}(x)=\overline{\operatorname{conv}}\left\{D\left(\varphi_{n}(x), r_{n}\right) \cap \Phi(x)\right\}, \quad x \in X .
$$

Отображение $\Phi_{n+1}: X \rightarrow B$ имеет непустые (по индукционному предположению) замкнутые выпуклые значения и полунепрерывно снизу в силу полунепрерывности снизу отображения $\Phi$ и непрерывности однозначного отображения $\varphi_{n}$. Тогда для непрерывной однозначной селекции $\varphi_{n+1}$ отображения $\Phi_{n+1}$ при всех $x \in X$ верны неравенства $\left\|\varphi_{n+1}(x)-\varphi_{n}(x)\right\| \leqslant r_{n}=q_{\alpha}^{n}(r)$ и в силу определения функции невьпуклости $\alpha_{\Phi(x)}$ множества $\Phi(x)$ получаем

$$
\operatorname{dist}\left(\varphi_{n+1}(x), \Phi(x)\right) \leqslant \alpha_{\Phi(x)}\left(r_{n}\right) \cdot r_{n}<\alpha\left(r_{n}\right) \cdot r_{n}=r_{n+1}=q_{\alpha}^{n+1}(r) .
$$

По условию теоремы функция $\alpha:(0, \infty) \rightarrow[0, \infty)$ является $G$-суммируемой, т.е. функциональный ряд $\sum_{n=0}^{\infty} q_{\alpha}^{n}(\cdot)$ поточечно сходится на $(0, \infty)$. В частности, сходится и числовой ряд $\sum_{n=0}^{\infty} q_{\alpha}^{n}(r)$. Значит, последовательность отображений $\left\{\varphi_{n}\right\}$ является фундаментальной в равномерной метрике и поэтому равномерно на $X$ 
сходится к некоторому непрерывному отображению $\varphi: X \rightarrow B$, которое и будет искомой непрерьвной селекцией отображения $\Phi$. При этом ясно, что

$$
\begin{aligned}
\|\varphi(x)-g(x)\| & =\left\|\varphi(x)-\varphi_{0}(x)\right\| \leqslant \sum_{n=0}^{\infty}\left\|\varphi_{n+1}(x)-\varphi_{n}(x)\right\| \\
& \leqslant \sum_{n=0}^{\infty} q_{\alpha}^{n}(r)=S_{\alpha}(r)<r+S_{\alpha}(r)=\beta(r) .
\end{aligned}
$$

Первый случай в теореме 6 доказан. Отметим, что здесь нам потребовалась сходимость ряда $\sum_{n=0}^{\infty} q_{\alpha}^{n}(t)$ в единственной точке $t=r$.

Случай 2. Рассмотрим случай произвольного непрерывного однозначного отображения $g: X \rightarrow B$. Выберем $r>1$ так, чтобы было непусто открытоемножество $G_{0}=\{x \in X \mid D(g(x), r) \cap \Phi(x) \neq \varnothing\}$ и положим

$$
G_{n}=\left\{x \in X \mid D\left(g(x), q_{\beta}^{n}(r)\right) \cap \Phi(x) \neq \varnothing\right\}
$$

Так как $\beta(r)=r+S_{\alpha}(r) \geqslant 2 r$, то из равенств $q_{\beta}^{1}(r)=\beta(r) \cdot r$ и $q_{\beta}^{n+1}(r)=\beta\left(q_{\beta}^{n}(r)\right)$. $q_{\beta}^{n}(r)$ и из неравенства $r>1$ следует, что числовая последовательность $\left\{q_{\beta}^{n}(r)\right\}$ монотонно стремится к бесконечности. Значит, $\varnothing \neq G_{0} \subset G_{1} \subset \cdots \subset G_{n} \subset \cdots$ и $\bigcup\left\{G_{n} \mid n \in \mathbb{N}\right\}=X$. Паракомпактность (даже счетная паракомпактность) пространства $X$ гарантирует тогда сушествование монотонно (по включению) возрастающей последовательности замкнутых подмножеств $A_{n} \subset G_{n}$, дающих в объединении все пространство $X$. Более того, можно считать семейство $\left\{A_{n}\right\}$ локально конечньм.

Применим теперь разобранньй вьше случай 1 к паракомпакту $X=A_{0}$, полунепрерывному снизу отображению $\Phi_{0}=\left.\Phi\right|_{A_{0}}$, выбранному выше числу $r>1$ и однозначной непрерывной $r$-селекции $g_{0}=\left.g\right|_{A_{0}}$ отображения $\Phi_{0}$. Получим на паракомпакте $A_{0}$ селекцию $\varphi_{0}$ отображения $\Phi_{0}$ такую, что

$$
\left\|\varphi_{0}(x)-g_{0}(x)\right\| \leqslant S_{\alpha}(r)<\beta(r)<r \cdot \beta(r)=q_{\beta}^{1}(r)
$$

Применим случай 1 к паракомпакту $X=A_{1}$, числу $q_{\beta}^{1}(r)$ и к однозначной непрерывной $q_{\beta}^{1}(r)$-селекции $g_{1}=\left.g\right|_{A_{1}}$ полунепрерывного снизу отображения $\Phi_{1}$, которое на $A_{0}$ совпадает с построенной выше селекцией $\varphi_{0}$, а на $A_{1} \backslash A_{0}$ совпадает с отображением $\Phi$. Если $x \in A_{0}$, то $\operatorname{dist}\left(g_{1}(x), \Phi_{1}(x)\right)=\left\|g_{0}(x)-\varphi_{0}(x)\right\|<q_{\beta}^{1}(r)$, если же $x \in A_{1} \backslash A_{0}$, то $\operatorname{dist}\left(g_{1}(x), \Phi_{1}(x)\right)=\operatorname{dist}(g(x), \Phi(x))<q_{\beta}^{1}(r)$ в силу условия $A_{1} \subset G_{1}=\left\{x \in X \mid D\left(g(x), q_{\beta}^{1}(r) \cap \Phi(x) \neq \varnothing\right\}\right.$. Значит, случай 1 действительно применим, и мы получаем на паракомпакте $A_{1}$ селекцию $\varphi_{1}$ отображения $\Phi_{1}$, которая на $A_{0}$ совпадает с $\varphi_{0}$ и такую, что

$$
\left\|\varphi_{1}(x)-g_{1}(x)\right\| \leqslant S_{\alpha}\left(q_{\beta}^{1}(r)\right)<\beta\left(q_{\beta}^{1}(r)\right)<q_{\beta}^{1}(r) \cdot \beta\left(q_{\beta}^{1}(r)\right)=q_{\beta}^{2}(r) .
$$

Продолжая по индукции, мы для каждого $x \in X$ определим некоторьй элемент $\varphi_{n(x)}=\varphi_{n(x)+1}=\cdots=\varphi(x) \in \Phi(x)$, где $n(x)=\min \left\{n \in \mathbb{N} \mid x \in A_{n}\right\}$. В силу 
локальной конечности семейства $\left\{A_{n}\right\}$ некоторая окрестность точки $x$ пересекается только с конечным числом элементов этого семейства, а значит, целиком содержится в некотором $A_{n}, n>n(x)$. Значит, отображение $\varphi$ в этой окрестности точки $x$ совпадает с непрерьвньп отображением $\varphi_{n}$ и поэтому само непрерывно в $x$. Отметим, что в отличие от случая 1 никакой явной оценки на расстояние между $g$ и селекцией $\varphi$ здесь получить не удается. Теорема 6 доказана.

Теорему 6 можно усилить, заменив условие равномерного мажорирования $\alpha_{\Phi(x)} \leqslant \alpha, x \in X$, условием локально равномерного мажорирования.

Теорема $6^{\prime}$. Пусть $\Phi: X \rightarrow B$ - полунепрерьвное снизу отображсение паракомпакта $X$ в банахово пространство $B$, и пусть для каждой точки $x \in X$ найдется ее окрестность $W(x)$ и найдется G-суммируемая функиия $\alpha_{x}$, строго мажсорирующая функиии невыпуклости всех значений $\Phi(z)$, $z \in W(x)$. Тогда существует непрерьвная однозначная селекиия отображения $\Phi$.

ДокаЗАТЕЛЬСТво ТЕОРЕмЫ 5. 1) Для фиксированного $\varepsilon>0$ числовой ряд $\sum_{n=0}^{\infty} q_{\alpha}^{n}(\varepsilon)$ сходится, где $\alpha(\cdot)$ - строгая $G$-суммируемая мажоранта функции невыпуклости множества $P$. Выберем номер $N \in \mathbb{N}$ так, чтобы $\sum_{n=N}^{\infty} q_{\alpha}^{n}(\varepsilon)<\varepsilon$ и положим $\delta=q_{\alpha}^{N}(\varepsilon)$. Так как функция $\alpha$ строго больше нуля, то и $\delta=q_{\alpha}^{N}(\varepsilon)>0$. Тогда $S_{\alpha}(\delta)=\sum_{n=0}^{\infty} q_{\alpha}^{n}\left(q_{\alpha}^{N}(\varepsilon)\right)=q_{\alpha}^{N}(\varepsilon)+q_{\alpha}^{N}(\varepsilon) \cdot \alpha\left(q_{\alpha}^{N}(\varepsilon)\right)+\cdots=\sum_{n=N}^{\infty} q_{\alpha}^{n}(\varepsilon)<\varepsilon$. Теперь на метризуемом, а значит, на паракомпактном пространстве $X=D_{\delta}(P)$ рассмотрим многозначное отображение $\Phi: X \rightarrow B$, которое каждую точку $x$ из $P$ переводит в себя и каждую точку $x$ из $X \backslash P$ переводит в $P$. Кроме того, рассмотрим тождественное отображение $g$ пространства $X$. Ясно, что $g$ есть непрерывная однозначная $\delta$-селекция отображения $\Phi$ и что функции невыпуклости всех значений отображения $\Phi$ строго мажорируются функцией $\alpha(\cdot)$. Применяем теорему 6 , случай 1 , и получаем непрерывную селекцию $\varphi$ отображения $\Phi$, которая автоматически будет ретракцией $X$ на $P$. Кроме того, $\|\varphi(x)-x\| \leqslant S_{\alpha}(\delta)<\varepsilon$. Значит, линейная гомотопия $f(x, t)=(1-t) g(x)+t \varphi(x), t \in[0,1]$, будет гомотопией $g$ и $\varphi$ внутри $\varepsilon$-окрестности $D_{\varepsilon}(P)$ множества $P$.

Утверждение 2) стандартно следует из теоремы 6, так как всякая задача о продолжении однозначного отображения есть частный случай некоторой задачи о нахождении непрерывной селекции, а последняя задача по теореме 6 имеет решение в классе паракомпактов. Теорема 5 доказана.

ДоКАЗАТЕЛЬСТво ТЕОРЕмЫ 4. Напомним, что всякое многозначное отображение $F$ метрического компакта $K$ в себя с замкнутьм графиком $\Gamma(F) \subset K \times K$ при любом $\varepsilon>0$, допускаюшее $\varepsilon$-аппроксимации $f_{\varepsilon}: K \rightarrow K$, которые имеют неподвижные точки $x_{\varepsilon}$, само имеет неподвижную точку. Такой точкой будет любая предельная точка последовательности $\left\{x_{\varepsilon_{n}}\right\}$ при $\varepsilon_{n} \rightarrow 0$. Последнее утверждение, в частности, справедливо для полунепрерывных сверху компактнозначных отображений. Так как по условию теоремы 4 компакт $K$ имеет свойство неподвижной точки для непрерывных однозначных отображений, то остается проверить $\varepsilon$-аппроксимируемость данного отображения $F$. Для этого по теореме [7], [8] достаточно проверить, что все значения отображения $F$ являются $U V^{\infty}$-подмножествами банахова пространства $B$, т.е. что для любой окрестности $U$ множества $F(x)$ найдется меньшая окрестность $V$ этого множества такая, что 
при любом $n \in \mathbb{N}$ всякое непрерывное отображение $n$-мерной сфферы $S^{n}$ в $V$ гомотопически тривиально как отображение $S^{n}$ в $U$. Так как множество $P=F(x)$ является компактом, то любая его окрестность $U$ содержит некоторую $\varepsilon$-окрестность $D_{\varepsilon}(P)$. По утверждению 1$)$ теоремы 5 выберем $\delta>0$ так, чтобы $\delta$-окрестность $D_{\delta}(P)=V$ множества $P$ ретрагировалась бы на $P$ внутри $D_{\varepsilon}(P) \subset U$. При этом такая ретракция гомотопна тождественному отображению $V$ на себя. Тогда и любой сфероид, лежащий в $V$, гомотопируется внутри $U$ в некоторый сфероид, лежаший в $P$. Последний сфероид стягивается по $P$ (а значит, и внутри $U$ ) в точку вместе со всем $P$ в соответствии с утверждением 2 ) теоремы 5 . Теорема 4 доказана.

\section{§3. Контролируемый отказ от выпуклости. Конечномерный случай}

Доказательство теоремы 8 напоминает доказательство теоремы 6, т.е. на первом шаге следует научиться улучшать $r$-селекции до настоящих селекций. Различие состоит в том, что в теореме 6 выпуклозначная теорема Майкла применима к отображениям

$$
\Phi_{k+1}(x)=\overline{\operatorname{conv}}\left\{D\left(\varphi_{k}(x), r_{k}\right) \cap \Phi(x)\right\}, \quad x \in X
$$

а к отображениям

$$
\Psi_{k+1}(x)=\overline{\operatorname{conv}}_{n+1}\left\{D\left(\varphi_{k}(x), r_{k}\right) \cap \Phi(x)\right\}, \quad x \in X,
$$

никакая известная селекционная теорема (выпуклозначная или же конечномерная), вообще говоря, не применима, так как операция $\overline{\operatorname{conv}}_{n+1}$ может нарушать порядок локальной связности.

ПримеР. Существует дискретное (значит, локально связное) множество $A$, для которого множество $\overline{\mathrm{conv}}_{2} A$ не локально связно.

ДоКАЗАТЕЛЬСТво. В гильбертовом пространстве $l_{2}$ со стандартным базисом $e_{1}, e_{2}, e_{3}, \ldots$ можно рассмотреть множество

$$
A=\bigcup\left\{(1 / n) e_{2 n-1} \pm e_{2 n} \mid n \in \mathbb{N}\right\}
$$

Тогда начало координат $\theta$ принадлежит $\overline{\operatorname{conv}}_{2} A$, а все достаточно малые окрестности точки $\theta$ состоят из $\theta$ и дизъюнктного объединения счетного числа отрезков. Действительно, точка

$$
x=(1-t) \cdot\left((1 / n) e_{2 n-1} \pm e_{2 n}\right)+t \cdot\left((1 / k) e_{2 k-1} \pm e_{2 k}\right)
$$

при $n \neq k$ есть сумма двух точек, одна из которых лежит на отрезке $\left[(1 / n) e_{2 n-1}+\right.$ $\left.(1 / k) e_{2 k-1}\right]$, а другая - на отрезке $\left[ \pm e_{2 n}, \pm e_{2 k}\right]$. Значит, расстояние от $x$ до $\theta$ больше $1 / 2$ при всех достаточно больших $n$ и $k$. В то же время при $n=k$ получаем, что $(1 / n) e_{2 n-1} \in \overline{\operatorname{conv}}_{2} A$ и поэтому $\theta \in \overline{\operatorname{conv}}_{2} A$.

Вернемся к доказательству теоремы 8. Во-первых, заметим, что достаточно научиться только исправлять $r$-селекции до настоящих селекций. Действительно, обозначим через $\Pi_{\alpha}^{n}(B)$ семейство всех непустых замкнутых подмножеств $P$ банахова пространства $B$, у которых функция невыпуклости $\alpha_{P}^{n}$ (в размерности $n$ ) 
мажорируется фиксированной функцией $\alpha:(0, \infty) \rightarrow[0, \infty), \alpha<1-0$. Ясно, что все одноточечные множества лежат в $\Pi_{\alpha}^{n}(B)$. Если мы докажем, что всякое полунепрерывное снизу отображение $\Phi: X \rightarrow \Pi_{\alpha}^{n}(B)$ метрического $n$-мерного компакта $X$ имеет непрерывную однозначную селекцию, то по обратной селекционной теореме Майкла [18] получим, что все элементы семейства $\Pi_{\alpha}^{n}(B)(n-1)$-связны, а само семейство $\Pi_{\alpha}^{n}(B)$ равностепенно локально $(n-1)$-связно. Тогда по прямой селекционной теореме Майкла [18] получим, что компакт $X$ можно заменить на паракомпакт $X$, т.е. докажем теорему 8 . Итак, будем считать, что $X$ есть $n$-мерный компакт. Рассмотрим числовую функцию $d(x)=\operatorname{dist}(\theta, \Phi(x)), \theta$ - начало координат в $B, x \in X$. Легко видеть, что $d(\cdot)$ - полунепрерывная сверху числовая функция на компакте $X$, и поэтому она ограничена сверху некоторой константой $r>0$. Другими словами, постоянное отображение $\varphi_{0} \equiv \theta$ есть непрерывная $r$-селекция отображения $\Phi$ и достаточно будет, начиная с $\varphi_{0}$, построить некоторую селекцию отображения $\Phi$. Подчеркнем, что селекции многозначных отображений обычно [18] ищу тся в виде предела равномерно сходящейся последовательности непрерывных $\varepsilon$-селекций ("внешние" приближения) или же [22], [23] последовательности $\delta$-непрерывных селекций ("внутренние" приближения) данного многозначного отображения. В теореме 8 применяется смешанная техника, и ответ строится как равномерный предел последовательности $\delta$-непрерывных $\varepsilon$-селекций; $\delta \rightarrow 0$, $\varepsilon \rightarrow 0$. Выделим в отдельное утверждение индуктивный переход от $r_{n}$-селекции $\varphi_{n}$ к некоторой $r_{n+1}$-селекции $\varphi_{n+1} \mathrm{c} r_{n+1}<r_{n}$.

Теорема 13 (улучшение селекций). Пусть $\alpha:(0, \infty) \rightarrow(0, \infty)-$ функиия, строго мажсрирующая функиии невыпуклости (в размерности $n$ ) всех значений полунепрерьвного снизу отображения $F: X \rightarrow B$-мерного паракомпакта $X$ в банахово пространство В. Пусть заданы положительные числа $r, \delta, \sigma$ и однозначная $\delta$-непрерывная $r$-селекция $f: X \rightarrow B$ отображения $F$. Тогда для $r^{*}=r+2 \delta+\sigma$ существует однозначное отображсение $f^{*}: X \rightarrow B$ такое, что:

а) $f^{*}$ есть $\alpha\left(r^{*}\right) \cdot r^{*}$-селекция отображсения $F$;

б) $\left\|f^{*}(x)-f(x)\right\|<r^{*}$ для всех $x \in X$;

в) $f^{*} \sigma$-непрерьвно.

ДоКАЗАТЕЛЬСТво. Опишем сначала конструкцию из шести пунктов.

1) Для каждого $x \in X$ произвольно выберем по точке $y(x)$ из пересечения $F(x) \cap$ $D(f(x), r)$.

2) Для каждого $x \in X$ произвольно выберем по окрестности $V(x)$, в которой для всех $x^{\prime}$ верно неравенство $\left\|f\left(x^{\prime}\right)-f(x)\right\|<2 \delta$.

3) Определим непустую окрестность $W(x)=V(x) \cap F^{-1}(D(y(x), \sigma / 3))$ точки $x$ и рассмотрим открытое покрытие $\omega=\{W(x)\}, x \in X$, паракомпакта $X$.

4) Пусть $\left\{U_{\gamma}\right\}, \gamma \in \Gamma$, - локально конечное открытое покрытие $X$ кратности $\leqslant n+1$, вписанное в покрытие $\omega$, и $\left\{e_{\gamma}\right\}, \gamma \in \Gamma,-$ соответствуюшее разбиение единицы, $\operatorname{supp}\left(e_{\gamma}\right) \subset U_{\gamma}$.

5) Для каждого индекса $\gamma \in \Gamma$ произвольно фиксируем точку $x_{\gamma} \in X$ так, чтобы $U_{\gamma} \subset W\left(x_{\gamma}\right)$, и произвольно фиксируем однозначную селекцию (не непрерывную) $s_{\gamma}: \operatorname{supp}\left(e_{\gamma}\right) \rightarrow B$ многозначного отображения, которое каждой 
точке $x^{\prime} \in \operatorname{supp}\left(e_{\gamma}\right) \subset U_{\gamma} \subset W\left(x_{\gamma}\right)$ ставит в соответствие непустое множество $F\left(x^{\prime}\right) \cap D\left(y\left(x_{\gamma}\right), \sigma / 3\right)$.

6) Определим $f^{*}$ равенством $f^{*}(x)=\sum e_{\gamma}(x) \cdot s_{\gamma}(x), \gamma \in \Gamma$.

Теперь проверим свойства а), б), в). Для этого стандартным образом рассмотрим множества $A(x)=\left\{\gamma \in \Gamma \mid e_{\gamma}(x)>0\right\}$ и $B(x)=\left\{\gamma \in \Gamma \mid x \in \operatorname{supp}\left(e_{\gamma}\right)\right\}$. Ясно, что $f^{*}(x)=\sum e_{\gamma}(x) \cdot s_{\gamma}(x), \gamma \in A(x)$ и что число элементов в $A(x)$ не превосходит $n+1$, см. п. 4$)$. Оценим теперь размер не более чем $(n+1)$-элементного подмножества $\left\{s_{\gamma}(x)\right\}, \gamma \in A(x)$, банахова пространства $B$ :

$$
\left\|s_{\gamma}(x)-f(x)\right\| \leqslant\left\|s_{\gamma}(x)-y\left(x_{\gamma}\right)\right\|+\left\|y\left(x_{\gamma}\right)-f\left(x_{\gamma}\right)\right\|+\left\|f\left(x_{\gamma}\right)-f(x)\right\| .
$$

Первое слагаемое в правой части менше $\sigma / 3$ (см. п. 5)), второе слагаемое меншше $r$ (см. п. 1)), а третье - меньше $2 \delta$, так как $x \in \operatorname{supp}\left(e_{\gamma}\right) \subset U_{\gamma} \subset W\left(x_{\gamma}\right) \subset V\left(x_{\gamma}\right)$. Следовательно, все точки $\left\{s_{\gamma}(x)\right\}, \gamma \in A(x)$, удалены от $f(x)$ менее чем на $r+$ $2 \delta+\sigma / 3<r+2 \delta+\sigma=r^{*}$, т.е. лежат в пересечении $F(x) \cap D\left(f(x), r^{*}\right)$. По определению функции невыпуклости (в размерности $n$ ) получаем, что

$$
\operatorname{dist}\left(f^{*}(x), F(x)\right) \leqslant \alpha_{F(x)}^{n}\left(r^{*}\right) \cdot r^{*}<\alpha\left(r^{*}\right) \cdot r^{*},
$$

что доказывает свойство а).

Свойство б) сразу следует из оценки

$$
\begin{aligned}
\left\|f^{*}(x)-f(x)\right\| & =\left\|\sum e_{\gamma}(x) \cdot\left(s_{\gamma}(x)-f(x)\right)\right\| \\
& \leqslant \max \left\{\left\|s_{\gamma}(x)-f(x)\right\| \mid \gamma \in A(x)\right\}<r^{*} .
\end{aligned}
$$

Для доказательства в) рассмотрим для $x \in X$ открытую окрестность

$$
G(x)=\left(\bigcap\left\{\operatorname{Int}\left(\operatorname{supp}\left(e_{\gamma}\right)\right) \mid \gamma \in A(x)\right\}\right) \backslash\left(\bigcup\left\{\operatorname{supp}\left(e_{\gamma}\right) \mid \gamma \notin B(x)\right\}\right) .
$$

Хорошо известно [22], [12], что в этой окрестности верны включения

$$
A(x) \subset A\left(x^{\prime}\right) \subset B\left(x^{\prime}\right) \subset B(x), \quad x^{\prime} \in G(x) .
$$

Тогда для $x^{\prime} \in G(x)$ получаем, что

$$
\begin{aligned}
f^{*}\left(x^{\prime}\right)-f^{*}(x)= & \sum_{\gamma \in A\left(x^{\prime}\right)} e_{\gamma}\left(x^{\prime}\right) \cdot s_{\gamma}\left(x^{\prime}\right)-\sum_{\gamma \in A(x)} e_{\gamma}(x) \cdot s_{\gamma}(x) \\
= & \sum_{\gamma \in A(x)}\left[e_{\gamma}\left(x^{\prime}\right)-e_{\gamma}(x)\right] \cdot s_{\gamma}(x) \\
& +\sum_{\gamma \in A(x)} e_{\gamma}\left(x^{\prime}\right) \cdot\left[s_{\gamma}\left(x^{\prime}\right)-s_{\gamma}(x)\right] \\
& +\sum_{\gamma \in A\left(x^{\prime}\right) \backslash A(x)} e_{\gamma}\left(x^{\prime}\right) \cdot s_{\gamma}\left(x^{\prime}\right) .
\end{aligned}
$$

Первое слагаемое есть сумма $\leqslant n+1$ непрерывных на $G(x)$ отображений, каждое из которых в точке $x$ обращается в нуль. Значит, в некоторой окрестности точки $x$ это слагаемое по норме меньше $\sigma / 6$. Норма второго слагаемого меньше $2 \sigma / 3$, так как точки $s_{\gamma}\left(x^{\prime}\right)$ и $s_{\gamma}(x)$ по построению (см. п. 5)) лежат в шаре $D\left(y\left(x_{\gamma}\right), \sigma / 3\right)$. Наконец, третье слагаемое по норме не превосходит $\sum_{\gamma \in B(x) \backslash A(x)} e_{\gamma}\left(x^{\prime}\right) \cdot\left\|s_{\gamma}\left(x^{\prime}\right)\right\|$. Последняя сумма есть сумма конечного числа непрерывных числовых функций, каждая из которых равна нулю в точке $x$. Значит, в некоторой окрестности точки $x$ норма третьего слагаемого меньше $\sigma / 6$. Свойство в) и теорема 13 об улучшении селекций доказаны. 
ДОКАЗАТЕЛЬСТВО ТЕОРЕМЫ 8. Искомую селекцию $\varphi$ отображения $\Phi$ построим как равномерный предел последовательности $\delta_{n}$-непрерывных $r_{n}$-селекций $\varphi_{n}: X \rightarrow B$ отображения $\Phi$ с $\delta_{n} \rightarrow 0, r_{n} \rightarrow 0$.

Шаг 0). Постоянное отображение $\varphi_{0} \equiv \theta$ есть непрерьвная $r$-селекция отображения $\Phi$ для некоторого $r>0$. Значит, $r_{0}=r$.

Пусть $\beta$ есть $G$-суммируемая строгая мажоранта для $\alpha^{+}$. Тогда для некоторого $\tau_{0}>0$ из неравенства $r_{0}<r^{*}<r_{0}+\tau_{0}$ следует неравенство $\alpha\left(r^{*}\right) \cdot r^{*}<\beta\left(r_{0}\right) \cdot r_{0}=$ $q_{\beta}^{1}\left(r_{0}\right)$. Выберем $\delta_{0}, \delta_{1}>0$ так, чтобы $r_{0}<r^{*}=r_{0}+2 \delta_{0}+\delta_{1}<r_{0}+\tau_{0}$.

Шаг 1). Применим теорему об улучшении селекций в ситуации, когда

$$
F=\Phi, \quad f=\varphi_{0}, \quad r=r_{0}, \quad \delta=\delta_{0}, \quad \sigma=\sigma_{1}, \quad r^{*}=r_{0}+2 \delta_{0}+\delta_{1} .
$$

Получим $\delta_{1}$-непрерьвную $\alpha\left(r^{*}\right) \cdot r^{*}$-селекцию (а значит, и $q_{\beta}^{1}\left(r_{0}\right)$-селекцию) $\varphi_{1}: X \rightarrow B$ отображения $\Phi$ такую, что $\left\|\varphi_{1}-\varphi_{0}\right\|<r^{*}<r_{0}+\tau_{0}$. Так как верхний предел справа у функции $\alpha$ в точке $r_{1}=q_{\beta}^{1}\left(r_{0}\right)$ меньше $\beta\left(r_{1}\right)$, то для некоторого $\tau_{1}>0$ из неравенства $r_{1}<r^{*}<r_{1}+\tau_{1}$ следует неравенство $\alpha\left(r^{*}\right) \cdot r^{*}<\beta\left(r_{1}\right) \cdot r_{1}=q_{\beta}^{2}\left(r_{0}\right)$. Если при этом оказалось, что $2 \delta_{1} \geqslant \tau_{1}$, то мы просто уменьшим $\delta_{1}$ в шаге 0$)$ так, чтобы $2 \delta_{1}<\tau_{1}$. Важно при этом, что степень разрывности $\delta_{1}$ отображения $\varphi_{1}$ по теореме об улучшении селекций не зависит от степени разрывности $\delta_{0}$ отображения $\varphi_{0}$. Выберем $\delta_{2}>0$ так, чтобы

$$
r_{1}<r^{*}=r_{1}+2 \delta_{1}+\delta_{2}<r_{1}+\tau_{1} .
$$

Шаг 2). Применим теорему об улучшении селекций в ситуации, когда $F=\Phi$, $f=\varphi_{1}, r=r_{1}=q_{\beta}^{1}\left(r_{0}\right), \delta=\delta_{1}, \sigma=\delta_{2}, r^{*}=r_{1}+2 \delta_{1}+\delta_{2}$. Получим $\delta_{2}$-непрерывную $\alpha\left(r^{*}\right) \cdot r^{*}$-селекцию (а значит, и $q_{\beta}^{2}\left(r_{0}\right)$-селекцию) $\varphi_{2}: X \rightarrow B$ отображения $\Phi$ такую, что $\left\|\varphi_{2}-\varphi_{1}\right\|<r^{*}<r_{1}+\tau_{1}$. Так как верхний предел справа у функции $\alpha$ в точке $r_{2}=q_{\beta}^{2}\left(r_{0}\right)$ меншше $\beta\left(r_{2}\right)$, то для некоторого $\tau_{2}>0$ из неравенства $r_{2}<r^{*}<r_{2}+\tau_{2}$ следует неравенство $\alpha\left(r^{*}\right) \cdot r^{*}<\beta\left(r_{2}\right) \cdot r_{2}=$ $q_{\beta}^{3}\left(r_{0}\right)$. Если при этом оказалось, что $2 \delta_{2} \geqslant \tau_{2}$, то мы просто уменшим $\delta_{2}$ в шаге 1) нужным образом, т.е. так, чтобы $2 \delta_{2}<\tau_{2}$. Важно при этом, что степень разрывности $\delta_{2}$ отображения $\varphi_{2}$ по теореме об улучшении селекций не зависит от степени разрывности $\delta_{1}$ отображения $\varphi_{1}$. Выберем $\delta_{3}>0$ так, чтобы

$$
r_{2}<r^{*}=r_{2}+2 \delta_{2}+\delta_{3}<r_{2}+\tau_{2} .
$$

Продолжив по индукции, мы получим последовательность отображений $\varphi_{n}: X \rightarrow B$ таких, что $\varphi_{n}$ есть $\delta_{n}$-непрерывная $q_{\beta}^{n}\left(r_{0}\right)$-селекция отображения $\Phi$ и при этом $\left\|\varphi_{n+1}-\varphi_{n}\right\|<q_{\beta}^{n}\left(r_{0}\right)+\tau_{n}, 2 \delta_{n}<\tau_{n}$ для всех натуральных $n$. Для окончания доказательства остается в вышеприведенной процедуре заранее положить, что $\sum \tau_{n}<\infty$. Теорема 8 доказана.

ДокАЗАТЕЛЬСТво тЕОРемы 9. По теореме 8 всякий компакт $F(x), x \in X$, есть абсолютный экстензор, а значит, и абсолютный ретракт, в размерности $n$. В частности, $F(x)$ есть ретракт компакта $K$, которьй по условию является ретрактом объемлюшего банахова пространства $B$. Значит, всякий компакт $F(x), x \in X$, есть абсолютньй ретракт в классе метрических пространств. Ясно, что тогда все 
значения $F(x), x \in X$, отображения $F$ являются $U V^{n-1}$-подмножествами $K$. По аппроксимационной теореме [8] $F$ имеет неподвижную точку.

Закончим этот параграф̆ примером, показывающим, что у $n$-мерного абсолютного ретракта $P$ с $\alpha_{P}^{n}<1-0$ может не быть хорошей оценки для общей функции невьпуклости $\alpha_{P}$. Рассмотрим график функции $z=\left(1-x^{2}-y^{2}\right)^{1 / 2}$, и пусть $P$ - ограничение этого графика на две стороны равностороннего треугольника, вписанного в окружность $\left\{(x, y, 0) \mid x^{2}+y^{2}=1\right\}$. Тогда прямой подсчет показьвает, что функция невыпуклости (в размерности 1$) \alpha_{P}^{1}$ всюду не превосходит некоторой константы $q<1$. В то же время ясно, что $\alpha_{P}^{2}(1+t)=1 /(1+t) \rightarrow 1$ при $t \rightarrow 0$.

\section{$\S 4$. Устойчивость множеств, функции} невыпуклости которых заметно меньше единицы

ДОКАЗАТЕЛЬСТВО ПРЕДЛОЖЕНИЯ 11. Для произвольных чисел $R, \varepsilon>0$ в евклидовой плоскости $\mathbb{R}^{2}$ рассмотрим три точки

$$
\begin{aligned}
A_{ \pm} & =( \pm(R+\varepsilon \cos \varphi), \varepsilon \sin \varphi), \\
B & =(0, q(R+\varepsilon \cos \varphi)+\varepsilon \sin \varphi),
\end{aligned}
$$

где $\cos \varphi=q /\left(1+q^{2}\right)^{1 / 2}$ и $\sin \varphi=1 /\left(1+q^{2}\right)^{1 / 2}$. Проведем через точки $B, A_{+}$и $A_{-}$ дугу окружности. Обозначим эту дугу $P$. Если $C_{ \pm}=( \pm R, 0)$, то длины отрезков $A_{+} C_{+}$и $A_{-} C_{-}$равны $\varepsilon$, т.е. точки $C_{ \pm}$лежат в $\bar{D}_{\varepsilon}(P)$. Ясно, что угол между $A_{+} C_{+}$(соответственно $A_{-} C_{-}$) и положительным (отрицательным) направлением оси абсцисс равен именно $\varphi$ и $\operatorname{ctg} \varphi=q$. Кроме того, $A_{+} C_{+}$(соответственно $A_{-} C_{-}$) перпендикулярен отрезку $A_{+} B$ (отрезку $A_{-} B$ ). Функция невыпуклости $\alpha_{P}$ дуги $P$ вычисляется сравнительно просто и максимум этой функции, равный $q=\left(y(B)-y\left(A_{+}\right)\right) / x\left(A_{+}\right)$, достигается при $r=x\left(A_{+}\right)$.

Обозначим через $Q$ замкнутую $\varepsilon$-окрестность множества $P$. По построению начало координат $\theta$ лежит в $\operatorname{conv}(Q)$. Ясно, что минимум расстояний $\|x\|, x \in P$, достигается при $x \in\left\{A_{-}, A_{+}, B\right\}$. Значит,

$$
\operatorname{dist}(\theta, P) \geqslant \min \left\{\theta B, \theta A_{+}\right\}=\min \left\{q R+\varepsilon\left(1+q^{2}\right)^{1 / 2},\left(R^{2}+\varepsilon^{2}+2 R \varepsilon \cos \varphi\right)^{1 / 2}\right\}
$$

и поэтому

$\operatorname{dist}(\theta, Q) \geqslant \operatorname{dist}(\theta, P)-\varepsilon \geqslant \min \left\{q R+\varepsilon\left(1+q^{2}\right)^{1 / 2},\left(R^{2}+\varepsilon^{2}+2 R \varepsilon \cos \varphi\right)^{1 / 2}\right\}-\varepsilon$.

Решив относительно $R$ два неравенства

$$
q R+\varepsilon\left(1+q^{2}\right)^{1 / 2}-\varepsilon \geqslant p R \text { и }\left(R^{2}+\varepsilon^{2}+2 R \varepsilon \cos \varphi\right)^{1 / 2}-\varepsilon \geqslant p R,
$$

получаем, что

$$
R \leqslant \varepsilon \cdot \frac{\left(1+q^{2}\right)^{1 / 2}-1}{p-q} \text { и } R \geqslant 2 \varepsilon \cdot \frac{p-\cos \varphi}{1-p^{2}} .
$$

При фиксированных $\varepsilon$ и $q$ и при $p \rightarrow q$ правая часть второго неравенства стремится к числу, а правая часть первого неравенства стремится к $+\infty$. Значит, у системы этих двух неравенств есть решения $R$ при всех $p$, достаточно близких к $q$. 
Итак, при фиксированном $q \in[0,1)$ для любых $\varepsilon>0$ и любых достаточно близких к $q$ чисел $p \in(q, 1)$ найдется $R>0$ так, что для функции невьпуклости $\varepsilon$-окрестности $Q$ множества $P=P(R, \varepsilon)$ верно неравенство $\alpha_{Q}(R) \cdot R \geqslant p R>q R$, в то время как $\alpha_{P}(\cdot) \leqslant q$. При этом, если число $p$ уже выбрано, то при $\varepsilon \rightarrow 0$ верно, что $R \rightarrow 0$ просто потому, что $R$ пропорционально $\varepsilon$.

Теперь при фиксированном $q \in[0,1)$ для всех $n \in \mathbb{N}$ построим описанньм выше образом множества $P_{n}=P\left(R_{n}, \varepsilon_{n}\right)$ так, чтобы $\sum \varepsilon_{n}<\infty$ и $q<p \leqslant p_{n}$. Разместим копии множеств $P_{n}$ на фиксированной прямой и на достаточно далеких расстояниях друг от друга: например, расстояния между $P_{n}$ и $P_{n+1}$ больше суммы диаметров множеств $P_{n}$ и $P_{n+1}$ и меньше удвоенной суммы этих диаметров. После этого объединим эти копии, добавив еше отрезки $\left[\left(A_{n}\right)_{+},\left(A_{n+1}\right)_{-}\right]$и предел последовательности $\left\{A_{n}\right\}$. Получим компактное подмножество $K$ евклидовой плоскости, которое $q$-паравыпукло (т.е. $\left.\alpha_{K}(\cdot) \leqslant q\right)$ и у которого никакая его замкнутая $\varepsilon_{n}$-окрестность $Q$ не является $p$-паравыпукльп множеством. Предложение 11 доказано.

Для доказательства теоремы 10 потребуются некоторые предварительные результаты. Всюду ниже мы фиксируем обозначение $R(E)$ для чебышевского радиуса подмножества $E$ банахова пространства $B$, т.е. для инфимума множества радиусов шаров, содержащих $E$.

Следующее упрощение при оценке функции невыпуклости $\alpha_{P}$ множества $P$ связано с тем, что в хороших банаховых пространствах при вычислении $\alpha_{P}$ в точке $r>0$ достаточно рассматривать не все точки симплекса $\operatorname{conv}\left\{x_{1}, \ldots, x_{n}\right\}$, $x_{i} \in P \cap D, D$ - шар радиуса $r$, а в точности одну точку этого симплекса. Именно, чебышевский центр этого симплекса. Напомним, что чебышевский центр $c(E)$ ограниченного подмножества $E$ банахова пространства $B$ - это центр замкнутого шара радиуса $R(E)$ в пространстве $B$, который содержит множество $E$. А. Л. Гаркави охарактеризовал те банаховы пространства $B$, в которых чебышевские центры ограниченных множеств всегда сушествуют, а также те банаховы пространства $B$, в которых у любого ограниченного множества существует единственный чебышевский центр. Оказывается, что (в последнем случае) это в точности равномерно выпуклые банаховы пространства [24].

ОПРЕДЕЛЕНИЕ $1^{\prime}$. Централъной функиией невыпуклости $\widehat{\alpha}_{P}(\cdot)$ замкнутого непустого подмножества $P$ равномерно выпуклого банахова пространства $B$ называется функция, которая каждому числу $r>0$ ставит в соответствие число

$$
\widehat{\alpha}_{P}(r)=\sup \{\operatorname{dist}(c(D \cap P), P) / r \mid D \text { - открытый шар радиуса } r\} .
$$

ЛЕмма 14. Пусть $(B,\|\cdot\|)$ - равномерно выпуклое банахово пространство. Тогда для любого замкнутого ограниченного подмнохсества $P \subset B$ эквивалентны условия:

а) функция невыпуклости $\alpha_{P}$ заметно меньше единицы;

б) иентральная функиия невыпуклости $\widehat{\alpha}_{P}$ заметно меньие единиць. 
ДоказАТЕЛЬство. Ясно, что $\widehat{\alpha}_{P} \leqslant \alpha_{P}$ и поэтому б) следует иза). Для доказательства обратной импликации выберем произвольно $n \in \mathbb{N}$, точки $x_{1}, x_{2}, \ldots, x_{n}$ из $P$ с $R=R\left(\left\{x_{1}, \ldots, x_{n}\right\}\right)$. Пусть $c=c\left(\left\{x_{1}, \ldots, x_{n}\right\}\right)$ - чебышевский центр множества $\left\{x_{1}, \ldots, x_{n}\right\}$. Можно считать, что $\left\|c-x_{i}\right\|=R$ : в противном случае $c$ лежит в одной из граней многогранника $\operatorname{conv}\left\{x_{1}, \ldots, x_{n}\right\}$ и можно перейти в меньшую размерность. Для произвольного $\delta \in(0,1)$ проведем замкнутые шары $D_{i}$ радиуса $(1-\delta) \cdot R$ с центрами в точках $x_{i} ; i=1,2, \ldots, n$. Рассмотрим произвольную точку $x \in \operatorname{conv}\left\{x_{1}, \ldots, x_{n}\right\}$. Мы хотим оценить расстояние $\operatorname{dist}(x, P)$ через уже имеюшуюся оценку $\operatorname{dist}(c, P) \leqslant \widehat{\alpha}_{P}(R) \cdot R$. Для точки $x$, отличной от $c$, возможны ровно два случая.

Случай 1 . Точка $x$ лежит в одном из шаров $D_{i}$. Тогда расстояние от $x$ до множества $P$ не превосходит $(1-\delta) \cdot R$.

Случай 2 . Точка $x$ лежит вне объединения шаров $D_{i}$. Замкнутые шары $\widehat{D}_{i}$ радиуса $R$, концентричные шарам $D_{i}$, покрывают многогранник $\operatorname{conv}\left\{x_{1}, \ldots, x_{n}\right\}$. Значит, для некоторого $i \leqslant n$ точка $x$ лежит в разности $\widehat{D}_{i} \backslash D_{i}$. И, более того, отрезок $[x, c]$ целиком лежит в этой разности в силу звездности относительно точки $c$ множества $\operatorname{conv}\left\{x_{1}, \ldots, x_{n}\right\} \backslash \cup\left\{D_{i} \mid 1 \leqslant i \leqslant n\right\}$. Но тогда $\|x-c\| \leqslant \varepsilon_{B}(\delta) \cdot R$, где $\varepsilon_{B}(\cdot)$ - функция, обратная к модулю равномерной выпуклости пространства $B$, и значит, $\operatorname{dist}(x, P) \leqslant \operatorname{dist}(c, P)+\|x-c\| \leqslant\left(\widehat{\alpha}_{P}(R)+\varepsilon_{B}(\delta)\right) \cdot R$.

Рассмотрим теперь при фиксированном $R$ следуюшее уравнение относительно переменной $\delta \in(0,1)$ :

$$
\widehat{\alpha}_{P}(R)+\varepsilon_{B}(\delta)=1-\delta .
$$

В силу монотонности и непрерьвности $\varepsilon_{B}(\cdot)$ у этого уравнения имеется единственное решение $\delta=\delta(R)$. Определим теперь функцию $\alpha:(0, \infty) \rightarrow[0,1)$ равенством $\alpha(R)=1-\delta(R)$. Тогда из рассмотренных выше случай 1 и 2 следует, что

$$
\operatorname{dist}(x, P) \leqslant \alpha(R) \cdot R, \quad x \in \operatorname{conv}\left\{x_{1}, \ldots, x_{n}\right\},
$$

т.е. что функция $\alpha$ мажорирует функцию невыпуклости $\alpha_{P}$ множества $P$. При этом, если $G$-суммируемая функция $\widehat{\beta}$ мажорирует центральную функцию невыпуклости $\widehat{\alpha}_{P}$ множества $P$, то функция $\beta:(0, \infty) \rightarrow[0,1)$, заданная равенством $\beta(R)=1-\delta^{\prime}(R)$, где $\delta^{\prime}(R)$ есть корень уравнения

$$
\widehat{\beta}(R)+\varepsilon_{B}(\delta)=1-\delta,
$$

будет $G$-суммируемой мажорантой функции $\alpha$, а значит, и функции невьпуклости $\alpha_{P}$ множества $P$. Лемма 15 доказана.

В доказательстве теоремы 10 решаюшим техническим моментом является предложение 15 , формализуюшее следуюшее, геометрически “очевидное”, наблюдение: если в треугольнике одна из сторон почти равна сумме двух других, то расстояния от вершин треугольника до его сторон малы, т.е. треугольник "вытянут".

ПрЕДЛОЖЕНИЕ 15 . Пусть $(B,\|\cdot\|)$ - равномерно выпуклое банахово пространство. Тогда для любого $0<\lambda \leqslant 1$ найдется непрерывная функция $\sigma_{\lambda}:(0, \infty) \times(0, \infty) \rightarrow(0,1)$ такая, ито:

(a) из неравенств $\|x-y\| \leqslant a, 0<\|y-z\|=b,\|x-z\| \geqslant \sigma_{\lambda}(a, b) \cdot a+b$ cледyem, ито $\operatorname{dist}(z, \operatorname{span}\{x, y\}) \leqslant \lambda b$; 
(в) для всякого $b>0$ верхний предел функиии $\sigma_{\lambda}(\cdot, b)$ в точке $a=0$ меньше единиць.

ДоКАЗАТЕЛЬСТВо ТЕОРЕМЫ 10. Пусть фиксированы функция $\alpha<1-0$ и число $\varepsilon>0$. Определим функцию $\beta:(0, \infty) \rightarrow[0,1)$ в точке $r$ равенством $\beta(r)=$ $\max \left\{\alpha(r+\varepsilon), \sigma_{\lambda}(r, \varepsilon)\right\}$, где $\sigma_{\lambda}-$ функция из предложения 15 , построенная для числа $\lambda=1-\alpha(r+\varepsilon) \in(0,1]$.

Пусть $P$ - множество, функция невыпуклости $\alpha_{P}$ которого мажорируется функцией $\alpha$. Докажем, что функция невьпуклости $\alpha_{Q}$ замкнутой $\varepsilon$-окрестности $Q$ множества $P$ мажорируется функцией $\beta$. Для этого рассмотрим произвольный открытьй шар $D$ радиуса $r$, пересекаюший множество $Q$. Пусть $y_{1}, y_{2}, \ldots, y_{n} \in D \cap P$, а $z_{1}, z_{2}, \ldots, z_{n}$ - точки из $P$, для которых $\left\|y_{i}-z_{i}\right\| \leqslant \varepsilon$. Нам следует оценить расстояние $\operatorname{dist}(x, Q)$ для точек $x$ многогранника conv $\left\{y_{1}, \ldots, y_{n}\right\}$. Если одно из расстояний $\left\|x-z_{i}\right\|$ не больше $\varepsilon$, то $x \in Q$ и оценивать нечего. Если же все эти расстояния больше $\varepsilon$, то, подвинув точки $y_{i}$ к точке $x$ по отрезкам, можно считать, что $\left\|y_{i}-z_{i}\right\|=\varepsilon$. Пусть $c$ - чебышевский центр многогранника $\operatorname{conv}\left\{y_{1}, \ldots, y_{n}\right\}$. По лемме 14 достаточно оценить расстояние от точки $с$ до множества $Q$. Возможны ровно два случая:

1) для некоторого $1 \leqslant i \leqslant n$ верно неравенство

$$
\left\|c-z_{i}\right\|<\sigma \cdot r+\varepsilon, \quad \text { где } \sigma=\sigma_{\lambda}(r, \varepsilon) ;
$$

2 ) для всех $1 \leqslant i \leqslant n$ верны неравенства

$$
\left\|c-z_{i}\right\| \geqslant \sigma \cdot r+\varepsilon .
$$

В случае 1$) \operatorname{dist}(c, Q)<\sigma r \leqslant \beta(r) \cdot r$, так как $z_{i} \in P$. В случае 2) применяем предложение 15 к треугольникам $\Delta c y_{i} z_{i}$ :

$$
\left\|c-y_{i}\right\| \leqslant r, \quad 0<\left\|y_{i}-z_{i}\right\|=\varepsilon, \quad\left\|c-z_{i}\right\| \geqslant \sigma_{\lambda}(r, \varepsilon) \cdot r+\varepsilon .
$$

Получаем, что $\operatorname{dist}\left(z_{i}, \operatorname{span}\left\{c, y_{i}\right\}\right) \leqslant \lambda \varepsilon$. Пусть $w_{i}-$ точка на прямой $\operatorname{span}\left\{c, y_{i}\right\}$, для которой $\left\|z_{i}-w_{i}\right\|=\operatorname{dist}\left(z_{i}, \operatorname{span}\left\{c, y_{i}\right\}\right) \leqslant \lambda \varepsilon \leqslant \varepsilon$. Тогда точка $y_{i}$ лежит на отрезке $\left[c, w_{i}\right]$ и, в частности, точка $c$ лежит в многограннике $\operatorname{conv}\left\{w_{1}, \ldots, w_{n}\right\}$. В силу выпуклости шаров найдется точка $v$ из многогранника $\operatorname{conv}\left\{z_{1}, \ldots, z_{n}\right\}$ такая, что $\|c-v\| \leqslant \lambda \varepsilon$. Точки $z_{1}, \ldots, z_{n}$ лежат во множестве $P$ и лежат в шаре $D^{\prime}$ радиуса $r+\varepsilon$, концентричном шару $D$. Теперь к этому шару $D^{\prime}$, множеству $P$, точкам $z_{1}, \ldots, z_{n}$ из пересечения $D^{\prime} \cap P$ и точке $v \in \operatorname{conv}\left\{z_{1}, \ldots, z_{n}\right\}$ применяем определение функции невыпуклости $\alpha_{P}$. Получаем, что

$$
\operatorname{dist}(v, P) \leqslant \alpha_{P}(r+\varepsilon) \cdot(r+\varepsilon)<\alpha(r+\varepsilon) \cdot(r+\varepsilon)
$$

и поэтому в силу выбора $\lambda=1-\alpha(r+\varepsilon)$ получаем, что

$$
\operatorname{dist}(c, P)<\lambda \varepsilon+\alpha(r+\varepsilon) \cdot(r+\varepsilon)=\varepsilon+\alpha(r+\varepsilon) \cdot r .
$$

Значит, $\operatorname{dist}(c, Q)<\alpha(r+\varepsilon) \cdot r \leqslant \beta(r) \cdot r$. Теорема 10 доказана.

Отметим, что формула $\beta(r)=\max \left\{\alpha(r+\varepsilon), \sigma_{\lambda}(r, \varepsilon)\right\}$ даже в случае константной функции $\alpha$ дает для $\beta$ функциональный, а не константный ответ. Другими словами, не удается вывести паравыпуклость $Q=\bar{D}_{\varepsilon}(P)$ из паравьпуклости $P$. 
ДОКАЗАТЕЛЬСТВО ПРЕДЛОЖЕНИЯ 15. ДЛя ПростотЫ ограничимся случаем гильбертова пространства $H=B$. Для любых положительных $a$ и $b$ положим $\sigma_{1}$ равным положительному корню уравнения $t a+b=\left(a^{2}+b^{2}\right)^{1 / 2}$ и положим $\sigma_{2}$ равным положительному корню уравнения

$$
t-\left(1-t^{2}\right)(a / 2 b)=\left(1-\lambda^{2}\right)^{1 / 2} .
$$

Более явньм образом, для $\tau=a / b$ получаем

$$
\sigma_{1}=\frac{\left(1+\tau^{2}\right)^{1 / 2}-1}{2 \tau} \text { и } \sigma_{2}=\frac{\left[1+\tau^{2}+2 \tau\left(1-\lambda^{2}\right)^{1 / 2}\right]^{1 / 2}-1}{\tau} .
$$

Далее по определению положим $\sigma_{\lambda}(a, b)=\max \left\{\sigma_{1}, \sigma_{2}\right\}$. Тогда непрерывность $\sigma_{\lambda}(\cdot, \cdot)$ следует из явного вида $\sigma_{1}$ и $\sigma_{2}$, а условие $\lim _{a \rightarrow 0} \sigma_{\lambda}(a, b)<1$ следует из $\sigma_{1} \rightarrow 0$ и $\sigma_{2} \rightarrow\left(1-\lambda^{2}\right)^{1 / 2}<1$ при $\tau \rightarrow 0$. Для завершения доказательства рассмотрим произвольный треугольник $\Delta x y z$ такой, что

$$
\|x-y\| \leqslant a, \quad 0<\|y-z\|=b, \quad\|x-z\| \geqslant \sigma_{\lambda}(a, b) \cdot a+b=\sigma \cdot a+b .
$$

Пусть $w$ - основание перпендикуляра, опушенного из точки $z$ на прямую $\operatorname{span}\{x, y\}$. Тогда $\|x-z\| \geqslant \sigma \cdot a+b \geqslant \sigma_{1} \cdot a+b=\left(a^{2}+b^{2}\right)^{1 / 2} \geqslant\left(\|x-y\|^{2}+\|y-z\|^{2}\right)^{1 / 2}$. Значит, угол $\psi=\angle z y x$ не меньше прямого, и точка $y$ лежит на отрезке $[x, w]$. Поэтому $a^{2}+b^{2}-2 a b \cdot \cos \psi \geqslant\|x-y\|^{2}+\|y-z\|^{2}-2\|x-y\| \cdot\|y-z\| \cdot \cos \psi=\|x-z\|^{2} \geqslant(\sigma \cdot a+b)^{2}$ и, значит, $-2 a b \cdot \cos \psi \geqslant 2 \sigma a b-a^{2}\left(1-\sigma^{2}\right)$. Так как функция $t \mapsto t-\left(1-t^{2}\right)(a / 2 b)$ монотонно возрастает при $t>0$, то из неравенства $\sigma \geqslant \sigma_{2}$ получаем, что

$$
-\cos \psi \geqslant \sigma_{2}-\left(1-\sigma_{2}^{2}\right)(a / 2 b)=\left(1-\lambda^{2}\right)^{1 / 2} .
$$

Следовательно, $\operatorname{dist}(z, \operatorname{span}\{x, y\})=\|z-w\|=\|y-z\| \cdot \sin \psi \leqslant \lambda b$.

Верен и аналог теоремы 10 для функций невыпуклости (в размерности $n$ ).

ТеОРема $10^{\prime}$. Пусть даны равномерно выпуклое банахово пространство $(B,\|\cdot\|)$, функиия $\alpha:(0, \infty) \rightarrow[0,1)$ заметно меньшая единицы и число $\varepsilon>0$. Тогда существует функция $\beta:(0, \infty) \rightarrow[0,1)$ заметно меньиая единицы такая, что для любого множества $P$ с $\alpha_{P}^{n} \leqslant \alpha$ функиия невьпуклости (в размерности $n)$ замкнутой $\varepsilon$-окрестности $\bar{D}_{\varepsilon}(P)$ множества $P$ не превосходит функиии $\beta$.

Для доказательства теоремы $10^{\prime}$ достаточно заметить, что в доказательстве теоремы 10 количество вершин $z_{i} \in D^{\prime} \cap P$ многогранника $\operatorname{conv}\left\{z_{1}, \ldots, z_{n}\right\}$ не превосходит количества вершин $y_{i} \in D \cap P$ многогранника conv $\left\{y_{1}, \ldots, y_{n}\right\}$.

ДоКАЗАТЕЛЬСТво тЕОРЕМЫ 12 . Использование теоремы $10^{\prime}$ и теоремы 8 показывает, что и все значения многозначного отображения $F$ и любые $\varepsilon$-окрестности этих значений являются абсолютньми экстензорами в размерности $n$. Следовательно, все значения $F(x), x \in X$, есть $U V^{n-1}$-подмножества банахова пространства $B$. Использование аппроксимационной теоремы $[25]$ завершает доказательство теоремы 12 . 


\section{Список литературы}

1. Schauder J. Der Fixpunktsatz in Funktionalraumen // Studia Math. 1930. V. 2. P. 171-180.

2. Kakutani S. A generalization of Brouwer's fixed point theorem // Duke Math. 1941. V. 8. P. 457-459.

3. Bohnenblust H., Karlin S. On a theorem of Ville. Contribution to the theory of games // Ann. of Math. Stud. 1950. P. 155-160.

4. Gliksberg I. A further generalization of the Kakutani fixed point theorem // Proc. Amer. Math. Soc. 1952. V. 3. P. 170-174.

5. Борисович Ю. Г., Гельман Б. Д., Мыикис А. Д., Обуховский В. В. Топологические методы в теории неподвижных точек многозначных отображений // УМН. 1980. Т. 35. №1. C. 59-126.

6. Борисович Ю. Г., Гельман Б. Д., Мыикис А. Д., Обуховский В. В. Многозначные отображения // Математический анализ. Т. 19. М.: ВИНИТИ, 1982. С. 127-231.

7. Gorniewcz L., Granas A., Kryszewsky W. On the homotopy method in the fixed point index theory of multivalued mappings of compact absolute neighborhood retracts // J. Math. Anal. Appl. 1991. V. 161. P. 457-473.

8. Gutev V. A fixed-point theorem for $U V^{n}$ usco maps // Proc. Amer. Math. Soc. 1996. V. 124. P. 945-952.

9. Michael E. Paraconvex sets // Scand. Math. 1959. V. 7. P. 372-376.

10. Repovs D., Semenov P. On paraconvexity of graphs of continuous functions // Set-Valued Anal. 1995. V. 3. P. 23-32.

11. Repovs D., Semenov P. On functions of nonconvexity for graphs of continuous functions // J. Math. Anal. Appl. 1995. V. 196. P. 1021-1029.

12. Семенов П. В. Функционально паравьпуклые множества // Матем. заметки. 1993. T. 54. №6. C. $74-81$.

13. Семенов П.В. О паравьпуклости звездноподобных множеств // Сиб. матем. журн. 1996. Т. 37. № 2. С. 399-405.

14. Гельман Б. Д. Обобщение теоремы Какутани о неподвижной точке для многозначных отображений // ДАН СССР. 1973. Т. 209. № 1. С. 53-57.

15. Гельман Б. Д. О некоторых классах сечений многозначных отображений // Применение топологии в современном анализе. Воронеж: Изд-во ВГУ, 1985. С. 42-62.

16. Aubin J.-P., Cellina A. Differential inclusions. Set-valued maps and viability theory // Grundlehren der Math. Wiss. V. 264. Berlin: Springer-Verlag, 1984.

17. Энгелькинг Р. Общая топология. М.: Мир, 1986.

18. Michael E. Continuous selections. I, II // Ann. of Math. 1956. V. 63. №2. P. 361-382; V. 64. № 3. P. 562-580.

19. Reich S. Some problems and results in fixed points theory // Contemp. Math. 1983. V. 21. P. 179-187.

20. Daffer P., Kaneko $H$. Fixed points of generalized contractive multivalued mappings // J. Math. Anal. Appl. 1995. V. 192. P. 655-666.

21. Борсук K. Теория ретрактов. М.: Мир, 1971.

22. Michael E. Convex structures and continuous selections // Canad. J. Math. 1959. V. 11. P. 556-575.

23. Чобан M. М. Многозначные отображения и борелевские множества // Труды ММО. 1970. T. 22. C. 229-250.

24. Гаркави A. Л. Наилучшие возможные сети и наилучшее возможное разбиение множеств в линейном нормированном пространстве // Изв. АН СССР. Сер. матем. 1962. Т. 26. C. $87-106$.

25. Щепин Е. В., Бродский Н. Б. Селекции фильтрованных многозначных отображений // Труды МИРАН. 1996. Т. 212. С. 218-239. 